\title{
Syringin prevents cardiac hypertrophy induced by pressure overload through the attenuation of autophagy
}

\author{
FANGFANG LI $^{1,2^{*}}$, NING ZHANG ${ }^{1,2^{*}}$, QINGQING WU ${ }^{1,2}$, YUAN YUAN ${ }^{1,2}$, ZHENG YANG $^{1,2}$, \\ MENGQIAO ZHOU ${ }^{1,2}$, JINXIU ZHU ${ }^{1,2}$ and QIZHU TANG ${ }^{1,2}$ \\ ${ }^{1}$ Department of Cardiology, Renmin Hospital of Wuhan University, \\ ${ }^{2}$ Cardiovascular Research Institute of Wuhan University, Wuhan, Hubei 430060, P.R. China
}

Received August 16, 2015; Accepted November 29, 2016

DOI: $10.3892 / \mathrm{ijmm} .2016 .2824$

\begin{abstract}
Syringin, extracted from Eleutherococcus senticosus, is a major biologically active component of Chinese herbs. Studies have certified the multiple pharmacological properties of syringin. However, the role of syringin in cardiac hypertrophy and the mechanisms involved remain unclear. In this study, aortic banding was performed on mice in order to induce cardiac hypertrophy, and the animals were then treated with syringin for 7 weeks. Echocardiography and catheter-based measurements of hemodynamic parameters were performed to evaluate cardiac function at 8 weeks following aortic banding. Morphological and pathological changes were also evaluated. Alterations in the expression levels of hypertrophy- and autophagy-related markers [atrial natriuretic peptide (ANP), $\beta$-myosin heavy chain MHC), $\alpha$-MHC, B-type natriuretic peptide (BNP), autophagy-related gene (ATG)5, ATG7, beclin 1, light chain 3 (LC3) A/B] were measured by reverse transcription-quantitative PCR and western blot analysis. The effects of syringin on cardiomyocyte hypertrophy induced by angiotensin II in H9c2 cells were also investigated. The results revealed that syringin attenuated cardiac hypertrophy induced by aortic banding via the activation of AMP-activated protein
\end{abstract}

Correspondence to: Professor Qizhu Tang, Department of Cardiology, Renmin Hospital of Wuhan University, 238 Jiefang Road, Wuhan, Hubei 430060, P.R. China

E-mail: qztang@126.com

*Contributed equally

Abbreviations: AMPK $\alpha$, AMP-activated protein kinase $\alpha$; AB, aortic banding; LVEDd, left ventricular end-diastolic diameter; IVSd, interventricular septum depth; H\&E, hematoxylin and eosin; WGA, wheat germ agglutinin; ANP, atrial natriuretic peptide; BNP, B-type natriuretic peptide; $\alpha$-MHC, $\alpha$-myosin heavy chain; $\beta$-MHC, $\beta$-myosin heavy chain; ATG, autophagy-related gene; LC3, light chain 3; GAPDH, glyceraldehyde-3-phosphate dehydrogenase; CSA, crosssectional area; FS\%, fractional shortening percentage; EF\%, ejection fraction percentage; Ang II, angiotensin II

Key words: syringin, hypertrophy, autophagy, AMP-activated protein kinase $\alpha$ kinase $\alpha$ (AMPK $\alpha$ ) and autophagy-related signaling pathways. Thus, we our data suggest that syringin possesses therapeutic potential to attenuate the progression of cardiac hypertrophy.

\section{Introduction}

Cardiac hypertrophy is characterized by the enlargement of cardiomyocytes and interstitial fibrisis (1-4). Hypertension, myocardial infarction and cardiomyopathy are often associated with interstitial fibrosis, contractile and diastolic dysfunction, and the re-expression of fetal cardiac genes, such as atrial natriuretic peptides (ANPs), brain natriuretic peptides or B-type natriuretic peptides (BNPs) and the $\beta$-myosin heavy chain (MHC), which can finally result in heart failure (5-8). In the initial stage, myocardial hypertrophy is an adaptive and compensatory process which maintains cardiac output; however, sustained cardiac hypertrophy leads to detrimental cardiac remodeling and heart failure, which is the main reason of morbidity and mortality worldwide $(1,2,5,6,8)$. New strategies to prevent or attenuate the enlargement of cardiomyocytes and cell apoptosis, and prevent the transition between adaptive hypertrophy and heart failure are necessary (1,9-11). Over the years, there have many efforts made to elucidate the molecular mechanisms of action of the intracellular signaling pathways involved in the progression of cardiac hypertrophy (1-8), in order to identify novel pharmacological agents with which to prevent heart failure.

Syringin $\left(\mathrm{C}_{17} \mathrm{H}_{24} \mathrm{O}_{9}\right.$, molecular weight, 372.37), also known as eleutheroside $\mathrm{B}$, is the major biologically active component extracted from Eleutherococcus senticosus (12-14). Various studies have certified the multiple pharmacological properties of syringin. Cui et al demonstrated that syringin decreased sleep latency and prolonged sleep duration in mice; the molecular mechanisms involved may be associated with the nitric oxide synthase (NOS)/nitric oxide (NO) pathway (13). Furthermore, there is evidence proving that syringin exerts remarkable anti-inflammatory and antioxidant effects, and possesses immunomodulatory properties (15-17). However, the effects of syringin on cardiac hypertrophy and its potential mechanisms of action have not yet been elucidated. The results of our study demonstrated that syringin attenuates the progression of cardiac hypertrophy induced by hypertrophic stimuli via the AMP-activated protein kinase $\alpha(\mathrm{AMPK} \alpha)$ and autophagy-related signaling pathways. 
The autophagy pathway is essential to myocardium homeostasis, and regulates cell survival and cell death pathways through the turnover of organelles and proteins (18-20). Nevertheless, under pathological conditions, the specific role of autophagy in cardiac geometry and function remains controversial. Zhu et al found that the autophagic response was activated by pressure overload, and they provided direct evidence that load triggered cardiac autophagy is a maladaptive response that contributes to the progression of heart failure (21). Another study demonstrated that multiple forms of stress, including pressure overload provoked an increase in autophagic activity in cardiomyocytes (22). On the contrary, other studies have suggested that autophagy activation may exert protective effects in cardiac hypertrophy and heart failure (23). Thus this study was designed to examine the role of syringin in the development of cardiac hypertrophy following pressure overload, and to elucidate the underlying mechanisms with a particular focus on autophagy.

\section{Materials and methods}

Chemicals. Syringin was obtained from Shanghai Winherb Medical S\&T Development Co., Ltd. (Shanghai, China). Highperformance liquid chromatography analysis was performed to confirm the purity of syringin ( $98 \%$; data not shown).

Animals. All animal experiments were performed in accordance with the institutional guidelines of the Animal Care and Use Committee of Renmin Hospital of Wuhan University, Wuhan, China. Male C57BL/6 mice weighing 23.5-27.5 g and aged 8-10 weeks were obtained from the Institute of Laboratory Animal Science, Chinese Academy of Medical Sciences and Peking Union Medical College (Beijing, China). The mice were housed in the Cardiovascular Research Institute of Wuhan University in an environment with controlled temperature and humidity. The mice were randomly divided into 5 groups of 20 mice each. Three of the groups were subjected to aortic banding $(\mathrm{AB})$ and the two other groups were subjected to sham surgery (Sham group). The aortic arch branch was exposed with a chest expander upon opening of the second and third intercostals by an incision. The vessel was ligated using a $26 \mathrm{G} / 27 \mathrm{G}$ syringe needle placed parallel above the vessel. Subsequent to the rapid withdrawal of the needle to achieve aortic constriction, the chest was closed in layers and a total of $0.1 \mathrm{ml} 0.5 \%$ bupivacaine (Sigma-Aldrich, St. Louis, MO, USA) was injected subcutaneously close to the edges of the skin incision to alleviate post-operative pain. The procedure for sham surgery was the same as that for $\mathrm{AB}$ but without vessel ligation. The mice in three of the treatment groups were gavaged with syringin at doses of $50 \mathrm{mg} / \mathrm{kg}$ body weight (L-syringin) or $100 \mathrm{mg} / \mathrm{kg}$ body weight (syringin) for 7 weeks. The mice in the remaining groups were gavaged with the same doses of normal saline (vehicle) for 7 weeks as a control. The 5 groups were designated as follows: sham + syringin, sham + vehicle, AB + vehicle, AB + L-syringin and $\mathrm{AB}+$ syringin. The mice were subjected to heart function determination by echocardiography and catheter-based measurements of hemodynamic parameters [heart weight/body weight (HW/BW; mg/g), lung weight/body weight (LW/BW; $\mathrm{mg} / \mathrm{g}$ ) and heart weight/tibia length (HW/TL; $\mathrm{mg} / \mathrm{ml}$ ) ratios; and the cross-sectional area of the cardiomyocytes (the areas of individual myocytes were calculated using Image Pro-Plus 6.0 software)] before being sacrificed (by cervical dislocation) at 8 weeks following surgery.

The mice were also subjected to heart function determination by echocardiography and catheter-based measurements of hemodynamic parameters before being sacrificed at 8 weeks following surgery. The mice were anesthetized with $1.5 \%$ isoflurane before performing echocardiography using a MyLab $^{\text {Tм }}$ 30CV (Esaote S.p.A., Genoa, Switzerland) and a $10 \mathrm{MHz}$ linear array ultrasound transducer (10). End-systole and end-diastole pressure were measured at the end of the left ventricular systole and diastole, respectively. Left ventricular end-diastolic diameter (LVEDd), interventricular septum depth (IVSd), fractional shortening percentage (FS\%) and ejection fraction percentage $(\mathrm{EF} \%)$ were also measured.

Echocardiography and hemodynamics. The mice were anesthetized with $1.5 \%$ isoflurane before being subjected to echocardiography using a MyLab ${ }^{\mathrm{TM}} 30 \mathrm{CV}$ cardiovascular ultrasound system (Esaote S.p.A., Genoa, Switzerland) and a $10 \mathrm{MHz}$ linear array ultrasound transducer. End-systolic and end-diastolic volume were measured at the end of the left ventricular systole and diastole, respectively. Left ventricular end-diastolic diameter (LVEDd), interventricular septum depth (IVSd) were measured from the left ventricle (LV) M-mode tracing with a sweep speed of $50 \mathrm{~mm} / \mathrm{sec}$ at the mid-papillary muscle level. The mice were also anesthetized with $1.5 \%$ isoflurane before being subjected to hemodynamic measurements. A microtip catheter transducer (SPR-839; Millar Instruments, Houston, TX, USA) was inserted into the right carotid artery and advanced into the left ventricle. The $\mathrm{dPdt}$ max and dPdt min end systolic volume and end-diastolic volume were determined. Data were continuously recorded with a Millar Pressure-Volume System (MPVS-400; Millar Instruments) and analyzed by PVAN analysis software.

Cell culture. The H9c2 cell line was acquired from the Cell Bank of Chinese Academy of Sciences (Shanghai, China). The cells were cultured in standard DMEM-basic, supplemented with $10 \%$ calf serum, $1 \%$ penicillin $(100 \mathrm{U} / \mathrm{ml})$ and streptomycin (100 mg/ml) (all from Gibco, Grand Island, NY, USA). The cells were incubated in a $\mathrm{CO}_{2}$ incubator (18 M; Sanyo, Osaka, Japan) with $5 \% \mathrm{CO} 2$ at $37^{\circ} \mathrm{C}$. Syringin was dissolved in dimethyl sulfoxide. The pharmaceuticals [angiotensin II (Ang II; Cat. no. A9525; Sigma-Aldrich), $2 \mu \mathrm{M}$; syringin, $15 \mu \mathrm{M}$ and rapamycin (Cat. no. 53123-88-9; Sigma-Aldrich), $100 \mathrm{nM}$ ] were added to the medium and the cells were incubated for $24 \mathrm{~h}$. Total RNA was then extracted from the H9c2 cells. The mRNA expression levels of ANP, $\beta$-MHC, $\alpha-\mathrm{MHC}$ and BNP were examined by reverse transcription-quantitative PCR (RT-qPCR). We characterized the cells by immunocytochemistry for cardiac $\alpha$-actin using $\alpha$-actin antibody (Cat. no. 2207266; EMD Millipore, Billerica, MA, USA) to assess cardiomyocyte hypertrophy. The cells also were detected by primary antibody to light chain 3 (LC3) A/B (Cat. no. 12741, Cell Signaling Technology Inc., Danvers, MA, USA) to assess autophagy.

Histological analysis. After the 8-week treatment period described above, the mouse hearts were rapidly excised, washed with phosphate-buffered saline, weighed and then immersed by 
Table I. Sequences of primers used for PCR.

\begin{tabular}{|c|c|c|}
\hline mRNA & Forward primer & Reverse primer \\
\hline ANP (rat) & 5'-AAAGCAAACTGAGGGCTCTGCTCG-3' & 5'-TTCGGTACCGGAAGCTGTTG CA-3' \\
\hline$\beta-\mathrm{MHC}$ (rat) & 5'-TCTGGACAGCTCCCCATTCT-3' & 5'-CAAGGCTAACCTGGAGAAGATG-3' \\
\hline$\alpha-\mathrm{MHC}$ (rat) & 5'-CAGAAAATGCACCATGAGGA-3' & 5'-TCAAGCATTCATATTTATTGTGGC-3' \\
\hline $\mathrm{BNP}$ (rat) & 5'-CAGCAGCTTCTGCATCGTGGAT-3' & 5'-TTCCTTAATCTGTCGCCGCTGG-3' \\
\hline GAPDH (rat) & 5'-GACATGCCGCCTGGAGAAAC-3' & 5'-AGCCCAGGATGCCCTTTAGT-3' \\
\hline ATG 5 (rat) & 5'-GGGACCTCCTGAATCTCACA-3' & 5'-CCAACAGGACAGCAGAGACA-3' \\
\hline ATG 7 (rat) & 5'-GTGTACGATCCCTGTAACCTAACCC-3' & 5'-CGAAAGCAGAGAACTTCAACAGACT-3' \\
\hline LC3 A/B (rat) & 5'-GACCCTCTACGATGCTGGTG-3' & 5'-TGCTGTCCTCAATGTCCTTCT-3' \\
\hline Beclin 1(rat) & 5'-GTGCTCCTGTGGAATGGAAT-3' & 5'-GCTGCACACAGTCCAGAAAA-3' \\
\hline ANP (mouse) & 5'-ACCTGCTAGACCACCTGGAG-3' & 5'-CCTTGGCTGTTATCTTCGGTACCGG-3' \\
\hline BNP (mouse) & 5'-GAGGTCACTCCTATCCTCTGG-3' & 5'-GCCATTTCCTCCGACTTTTCTC-3' \\
\hline$\alpha-\mathrm{MHC}$ (mouse) & 5'-GTCCAAGTTCCGCAAGGT-3' & 5'-AGGGTCTGCTGGAGAGGTTA-3' \\
\hline$\beta-\mathrm{MHC}$ (mouse) & 5'-CCGAGTCCCAGGTCAACAA-3' & 5'-CTTCACGGGCACCCTTGGA-3' \\
\hline GAPDH (mouse) & 5'-ACTCCACTCACGGCAAATTC-3' & 5'-TCTCCATGGTGGTGAAGACA-3' \\
\hline ATG 5 (mouse) & 5'-ATATCAGACCACGACGGAGCG-3' & 5'-CAGCATTGGCTCTATCCCGTG-3' \\
\hline ATG 7 (mouse) & 5'-CAGTTGGCTAGCTGTGTAA-3' & 5'-GGCTATCGACCCACTTGGA-3' \\
\hline LC3 A/B (mouse) & 5'-GCCATGGATCAACTATGG-3' & 5'-GGCACCTCACGGATCGGTA-3' \\
\hline Beclin 1 (mouse) & 5'-CTGGCCAGCAGCAGTTAGAC-3' & 5'-TCACGCTCCTCCGACGTCAA-3' \\
\hline
\end{tabular}

ANP, atrial natriuretic peptide; MHC, myosin heavy chain; BNP, brain natriuretic peptide; GAPDH, glyceraldehyde-3-phosphate dehydrogenase; ATG, autophagy-related protein; LC3, light chain 3.

perfusion with $10 \%$ neutral buffered formalin. To visualize the left and right ventricles, the hearts were cut transversely. Slides were prepared for hematoxylin and eosin (H\&E) staining for morphological evaluation. The slides were then visualized and photographed under a light microscope (Olympus FSX100; Olympus Corp., Tokyo, Japan). The cross-sections of myocytes stained with fluorescein isothiocyanate-conjugated wheat germ agglutinin (WGA; W849; Invitrogen, Carlsbad, CA, USA) to visualize membranes and DAPI to visualize nuclei were assessed. The areas of individual myocytes were calculated using Image Pro-Plus 6.0 software, a quantitative digital image analysis system.

$R T-q P C R$. The mRNA levels of the hypertrophic and autophagy markers, ANP, BNP, $\alpha$-MHC, $\beta$-MHC, autophagy-related gene (ATG)5, ATG7, beclin 1, LC3 A/B and glyceraldehyde3 -phosphate dehydrogenase (GAPDH) were analyzed by RT-qPCR. Total RNA was extracted from the cardiac tissues or H9c2 cells using TRIzol reagent following the manufacturer's instructions (Invitrogen). The RNA purities were evaluated based on the OD260/OD280 ratios detected with the SmartSpec Plus Spectrophotometer (Bio-Rad, Hercules, CA, USA). The mRNA was reverse transcribed into cDNA using oligo(dT) primers and the Transcriptor First Strand cDNA Synthesis kit (Roche, Basel, Switzerland). The primers used are listed in Table I. PCR amplifications were quantified using the LightCycler 480 SYBR-Green I Master Mix (Roche) in the LightCycler ${ }^{\circledR} 480$ Real-Time Quantitative PCR System (Roche). Briefy, subsequent to a 5-min initial denaturation at $95^{\circ} \mathrm{C}$, a total of 42 primer-extension cycles were conducted. Each cycle consisted of a 10 -sec denaturation step at $95^{\circ} \mathrm{C}$, a 20 -sec annealing step at $60^{\circ} \mathrm{C}$ and a $20-\sec$ incubation at $72^{\circ} \mathrm{C}$ for extension. A final extension step was thene conducted at $72^{\circ} \mathrm{C}$ for $10 \mathrm{~min}$. The double standard curve was used to quantify the PCR results. The results were normalized to the mRNA expression of glyceraldehydes-3-phosphate dehydrogenase (GAPDH).

Western blot analysis. Ice-cold radioimmunoprecipitation assay buffer was used to extract protein from tissue homogenates or cell lysates. Protein quantification was carried out using the Pierce BCA protein assay kit (Thermo Fisher Scientific, Waltham, MA, USA). The extracted protein was subjected to SDS-PAGE, after which the proteins were transferred to Immobilon-FL Transfer Membranes (Merck Millipore, Billerica, MA, USA) and incubated with various primary antibodies for $24 \mathrm{~h}$ in $4^{\circ} \mathrm{C}$. The primary antibodies were the following: GAPDH (Cat. no. sc-25778; Santa Cruz Biotechnology, Inc. Dallas, TX, USA), p-AMPKa (Cat. no. 2535; Cell Signaling Technology, Inc.), AMPK $\alpha$ (Cat. no. 2603P; Cell Signaling Technology, Inc. Danvers, MA, USA); ATG7 (Cat. no. 2631S; Cell Signaling Technology, Inc.), beclin 1 (Cat. no. ab55878; Abcam, Cambridge, UK), p62 (Cat. no. ab91526; Abcam), LC3 A/B (Cat. no. 12741; Cell Signaling Technology, Inc.). Following the removal of the primary antibody, the blots were incubated with the corresponding peroxidase-conjugated secondary antibodies [IRdye 800CW-conjugated goat antirabbit immunoglobulin (Ig)G (Cat. no. 926-32211; LI-COR Biosciences, Lincoln, NE, USA) and IRdye $800 \mathrm{CW}$-conjugated goat anti-mouse IgG (Cat. no. 926-32210; LI-COR Biosciences)] for $2 \mathrm{~h}$. The blots were then scanned using a two-color infrared imaging system (Odyssey, Danvers, MA, USA). Target protein expression levels were normalized to GAPDH protein. 
A

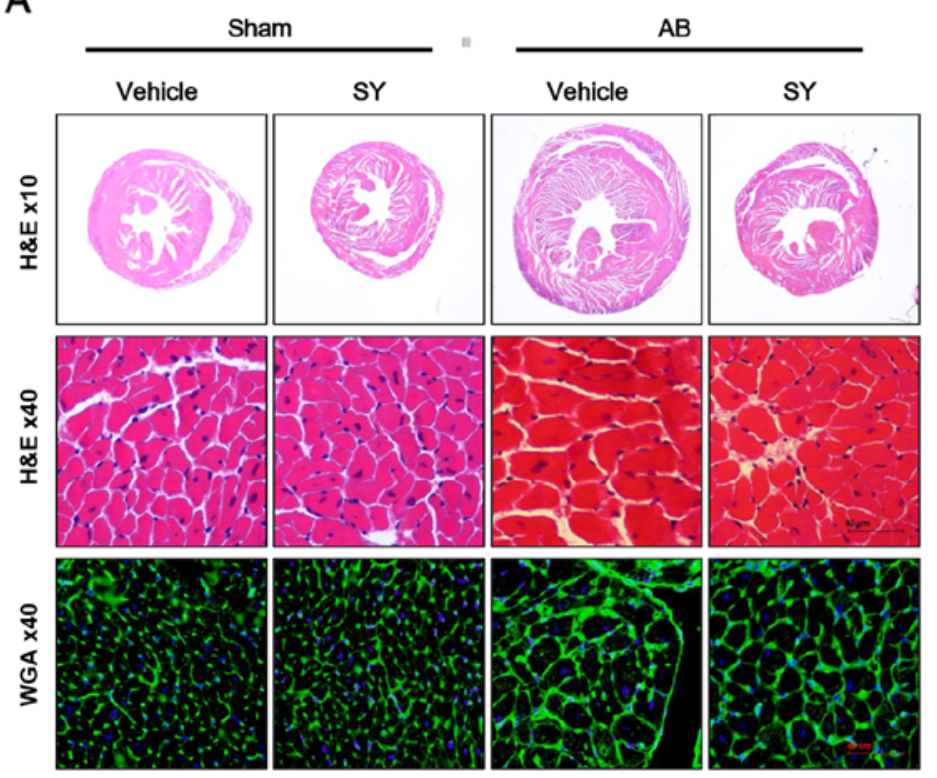

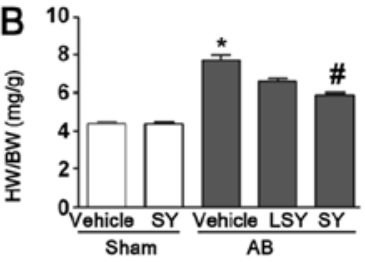
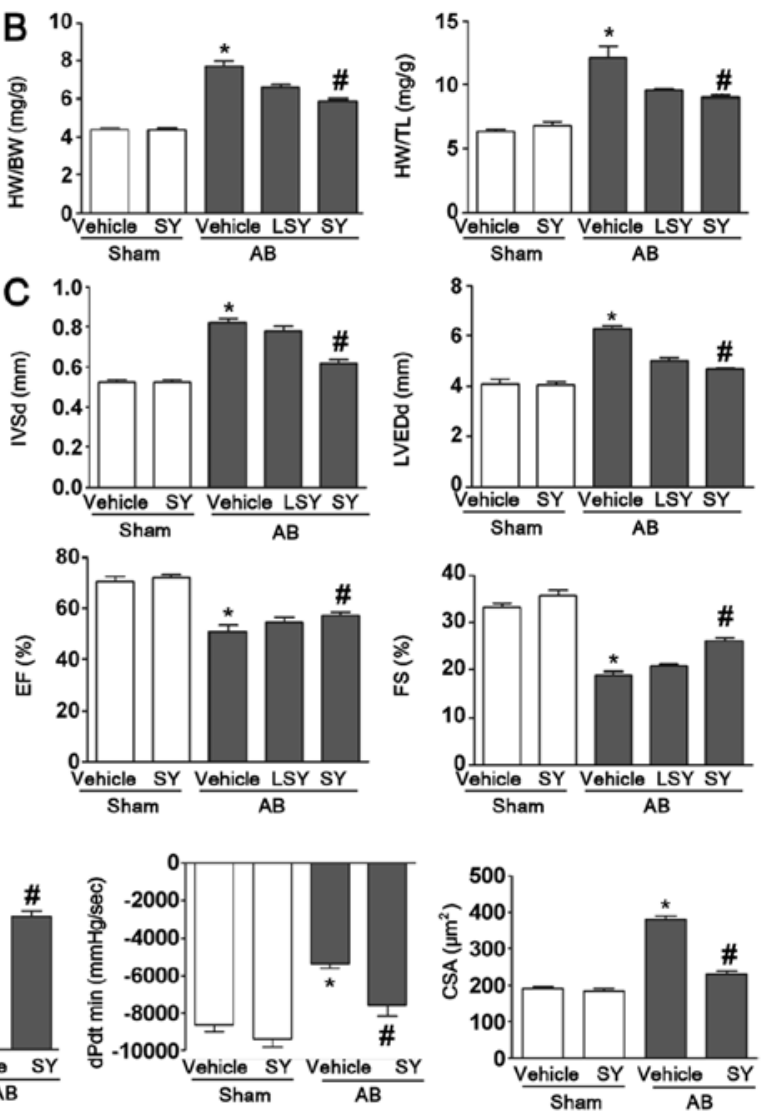

E
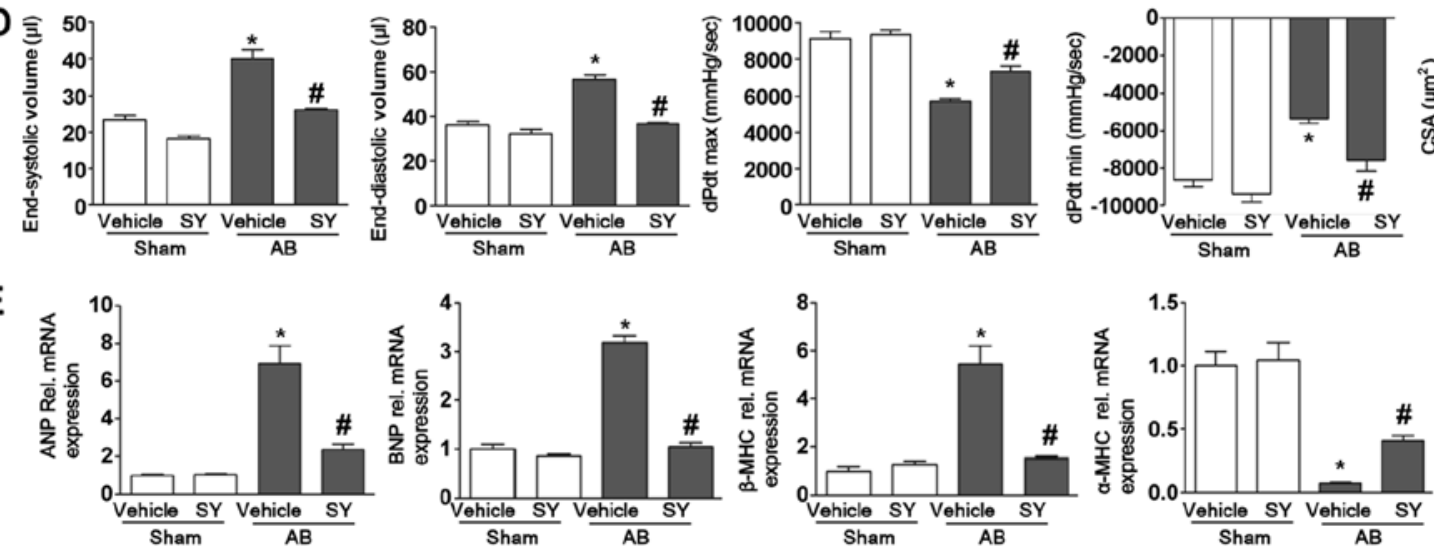

Figure 1. Syringin protects against cardiac hypertrophy. (A) Hematoxylin and eosin (H\&E) staining and fluorescein isothiocyanate-labeled wheat germ agglutinin (WGA) staining of sham-operated mice (Sham) and mice subjected to aortic banding (AB) at 8 weeks post-surgery. (B) Statistical results of the heart weight/ body weight $(\mathrm{HW} / \mathrm{BW})$ ratio and heart weight/tibial length (HW/TL) ratio. (C) Syringin ameliorated cardiac function as revealed by the parameter of left ventricular end-diastolic diameter (LVEDd), interventricular septum depth (IVSd), fractional shortening percentage (FS\%) and ejection fraction percentage (EF\%). (D) Syringin led to the hemodynamic parameters reverting back to normal levels and the myocyte cross-sectional areas decreasing. (E) Expression levels of atrial natriuretic peptide (ANP), B-type natriuretic peptide (BNP), $\beta$-myosin heavy chain (MHC), and $\alpha$-MHC induced by AB were determined by RT-qPCR. LSY, syringin $50 \mathrm{mg} / \mathrm{kg}$; SY, syringin $100 \mathrm{mg} / \mathrm{kg}$. ${ }^{*} \mathrm{p}<0.05$ compared with the corresponding sham-operated group; ${ }^{*} \mathrm{p}<0.05 \mathrm{vs}$. the $\mathrm{AB}+$ vehicle group.

Statistical analysis. Data were analyzed using SPSS 16.0 (SPSS, Inc., Chicago, IL, USA). Differences among the groups were determined by one-way ANOVA, and a value of $\mathrm{p}<0.05$ was considered to indicate a statistically significant difference.

\section{Results}

Syringin attenuates cardiac hypertrophy induced by pressure overload in mice. To determine whether syringin attenuates the hypertrophic response to pressure overload, mice were administered syringin after 7 days of $A B$ or sham surgery. The administration of syringin markedly decreased the heart weight/body weight ratio (HW/BW), heart weight/tibial length ratio (HW/TL) and the cross-sectional area (CSA) of the cardiomyocytes. As shown in Fig. 1, the protective effects of syringin on cardiac hypertrophy was confirmed by morphological anal- ysis, H\&E staining and WGA staining. The results of $\mathrm{H} \& \mathrm{E}$ and WGA staining revealed that syringin decreased cardiac mass and the myocyte cross-sectional area induced by pressure overload. The results of echocardiographic and pressure-volume loop analyses revealed that the LVEDd and IVSd increased, and the FS\% and $\mathrm{EF} \%$ decreased in the vehicle-treated mice following AB. Pressure overload significantly increased the LV mass and exacerbated cardiac function in the vehicle-treated group; however, treatment with syringin attenuated cardiac function and chamber dilation. The vehicle-treated mice exhibited ventricular dysfunction 8 weeks after $\mathrm{AB}$, as well as an increase in diastolic blood pressure and a decrease in systolic and diastolic functions. Syringin attenuated the dysfunction of the hemodynamic parameters, $\mathrm{dP} / \mathrm{dt} \max$ and $\mathrm{dP} / \mathrm{dt} \min$. In addition, the expression levels of hypertrophic markers, such as ANP, BNP and $\beta$-MHC significantly increased following AB. 

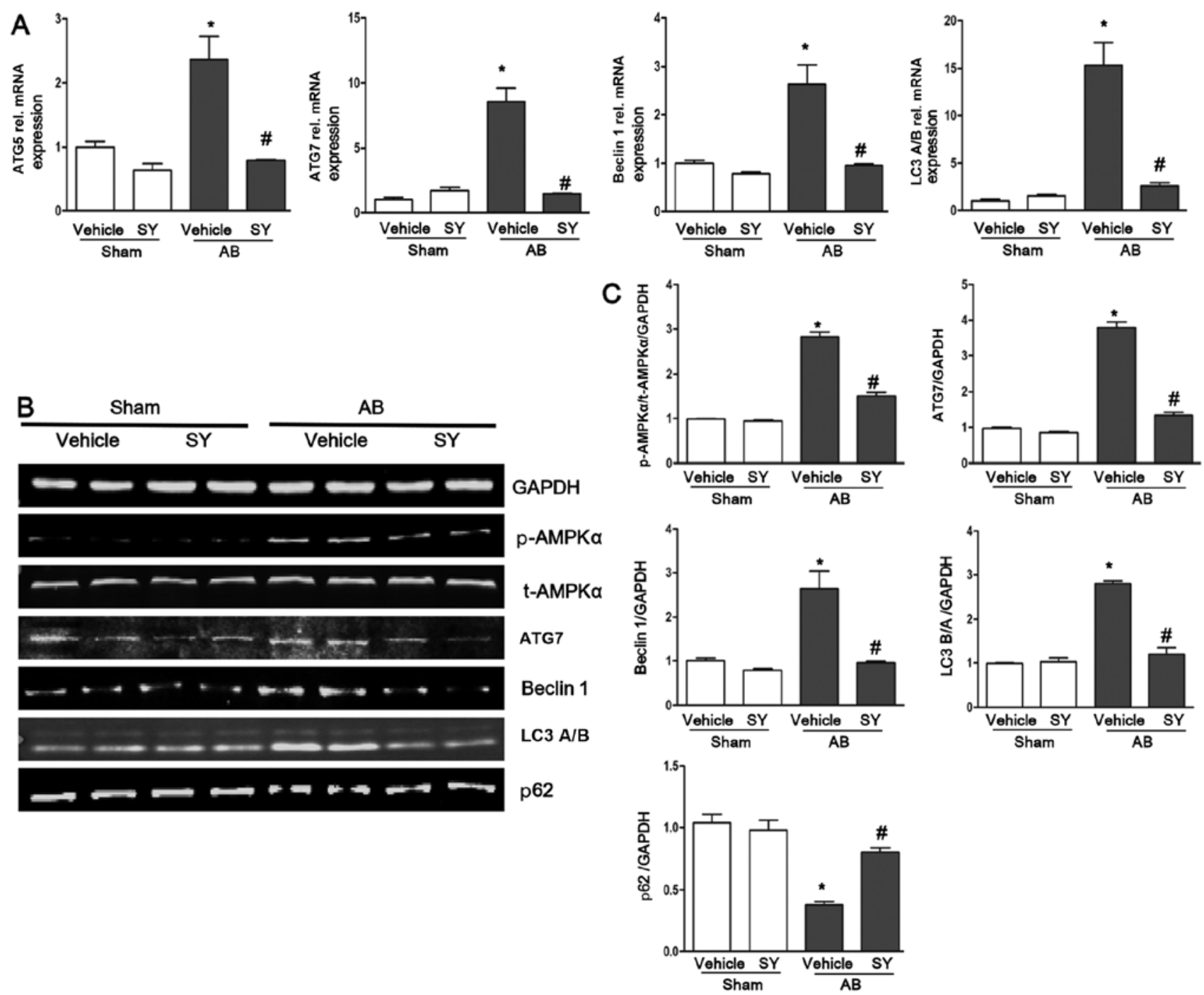

Figure 2. Syringin affects the AMP-activated protein kinase $\alpha$ (AMPK $\alpha$ ) and autophagy-related signaling pathways. (A) Expression levels of autophagy-related gene (ATG)5, ATG7, beclin 1 and light chain 3 (LC3) A/B induced by AB were determined by reverse transcription-polymerase chain reaction analysis. (B) Representative blots of p-AMPKa, ATG7, beclin 1, p62 and LC3 A/B in the heart tissues of mice. (C) Quantitative results. "p $<0.05$ compared with the corresponding sham-operated (Sham) group; ${ }^{\#} \mathrm{p}<0.05$ vs. the $\mathrm{AB}+$ vehicle group. $\mathrm{AB}$, aortic banding; $\mathrm{p}$, phosphorylation; $\mathrm{t}$, total; $\mathrm{SY}$, syringin $100 \mathrm{mg} / \mathrm{kg}$.

However, the administration of syringin decreased the expression levels of hypertrophic markers. In addition, the expression of $\alpha$-MHC decreased following AB and was elevated by the administration of syringin.

Syringin alleviates cardiac autophagy induced by pressure overload. The expression levels of the autophagy markers, ATG5, ATG7, beclin 1 and LC3 A/B, were detected by RT-qPCR in the present study. The results revealed that the expression levels of ATG5, ATG7, beclin 1 and LC3 A/B significantly increased following $\mathrm{AB}$, but were attenuated by the administration of syringin as shown in Fig. 2. We examined the protein expression levels of ATG7, beclin 1, p62 and LC3 A/B by western blot analysis. We found that the protein expression levels of ATG7 and beclin 1 were higher in the vehicle-treated mice than in the syringin-treated mice in response to $\mathrm{AB}$. The ratio of $\mathrm{LC} 3 \mathrm{~B} / \mathrm{A}$ was elevated following $\mathrm{AB}$ and was attenuated by the administration of syringin. In addition, p62 was found to be in inverse proportion to the ATG7 and beclin 1 (Fig. 2). These findings illustrated that syringin alleviated cardiac autophagy induced by pressure overload. To examine the molecular mechanisms responsible for the protective effects of syringin against the autophagy response, we examined the activation of AMPK $\alpha$. We found that AMPK $\alpha$ was significantly activated in the mice subjected to AB. The administration of syringin decreasesd the phosphorylation levels of AMPK $\alpha$ (Fig. 2).

Protective effects of syringin on cardiomyocyte hypertrophy in H9C2 cells. In in vitro experiments, the mRNA expression levels of hypertrophic markers, including ANP, BNP and $\beta$-MHC, were increased following stimulation with Ang II $(2 \mu \mathrm{M}, 24 \mathrm{~h})$. The levels of $\alpha-\mathrm{MHC}$ were decreased following stimulation with Ang II. Treatment with syringin $(15 \mu \mathrm{M})$ resulted in a marked decrease in the expression levels of hypertrophic markers and an inrease in the levels of $\alpha$-MHC. The cardiomyocytes were examined by immunocytochemistry using primary antibodies to cardiac $\alpha$-actin to assess cardiomyocyte hypertrophy. Thye H9c2 cells exposed to Ang II exhibited an enlarged cell surface area as compared with those treated with syringin $(15 \mu \mathrm{M})$. As 


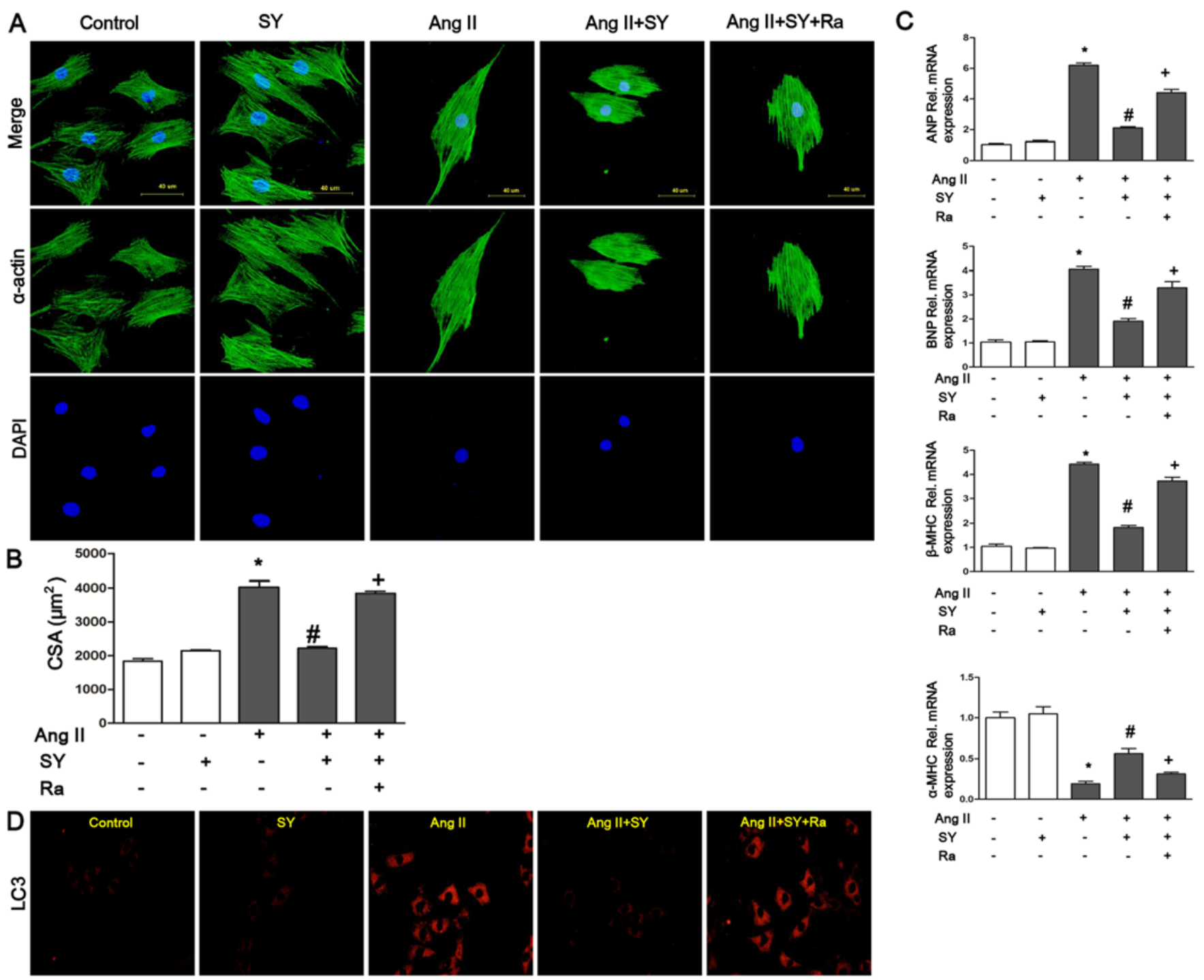

Figure 3. Syringin attenuates hypertrophy and autophagy in H9c2 cardiomyocytes induced by angiotensin II (Ang II). (A) Representative images of cardiomyocytes showing the inhibitory effect of syringin on cardiomyocyte enlargement in response to Ang II. (B) Quantification of cell cross-sectional areas by measuring 100 random cells. (C) RT-qPCR analysis of the mRNA levels of the hypertrophy markers, atrial natriuretic peptide (ANP), B-type natriuretic peptide (BNP), $\beta$-myosin heavy chain (MHC) and $\alpha$-MHC. (D) The cardiomyocytes stained by immunofluorescence for light chain 3 (LC3) in the different groups. "p<0.05 compared with the corresponding control group; ${ }^{*} \mathrm{p}<0.05$ vs. the Ang II group; ${ }^{+} \mathrm{p}<0.05$ vs. the Ang II + SY group. SY, syringin $15 \mu \mathrm{M} ; \mathrm{Ra}$, rapamycin $100 \mathrm{nM}$.

shown in Fig. 3, syringin alleviated cardiomyocyte hypertrophy induced by Ang II. The quantification of the cell CSA (Fig. 3B), indicated that the enlarged cell surface area induced by Ang II was significantly reduced by treatment with syringin; however, when rapamycin was added to the medium, the effect of syringin was reversed. Furthermore, syringin reduced the protein expression levels of ATG7 and beclin 1 induced by Ang II and elevated the levels of p62 (Fig. 4). To explore the molecular mechanisms through which syringin exerts its anti-hypertrophy and anti-autophagy effects, rapamycin, an autophagy agonist, was used in further experiments. Treatment of the cells with rapamycin attenauted the protective effects of syringin on cardiomyocytes. Treatment with rapamycin increased the levels of ANP, BNP and $\beta$-MHC, and decreased the levels of $\alpha$-MHC. We found that treatment of the H9c 2 cells with syringin decreased the single cardiomyocyte area and the levels of hypertrophic markers. In order to examine the role of autophagy, the cells were examined by immunofluorescence using primary antibodies to LC3. As shown in Fig. 3D, syringin decreased the expresstion of LC3. However, rapamycin attenuated this effect. Thus, our data prove that the protective effects of syringin against cardiac hypertrophy are closely related to autophagy.

\section{Discussion}

Syringin is a major biologically active component extracted from Eleutherococcus senticosus in Chinese herbs and possessed several biological functions, including anti-inflammatory, immunomodulatory actions and exerts antioxidant effects $(13,15-17,24)$. However, whether syringin has an effect on cardiac hypertrophy remains unclear. In this study, we found that syringin attenuated cardiac remodeling induced by pressure overload. The beneficial effects of syringin may be mediated by inhibition of the AMPK $\alpha$ and autophagy-related signaling pathways. 

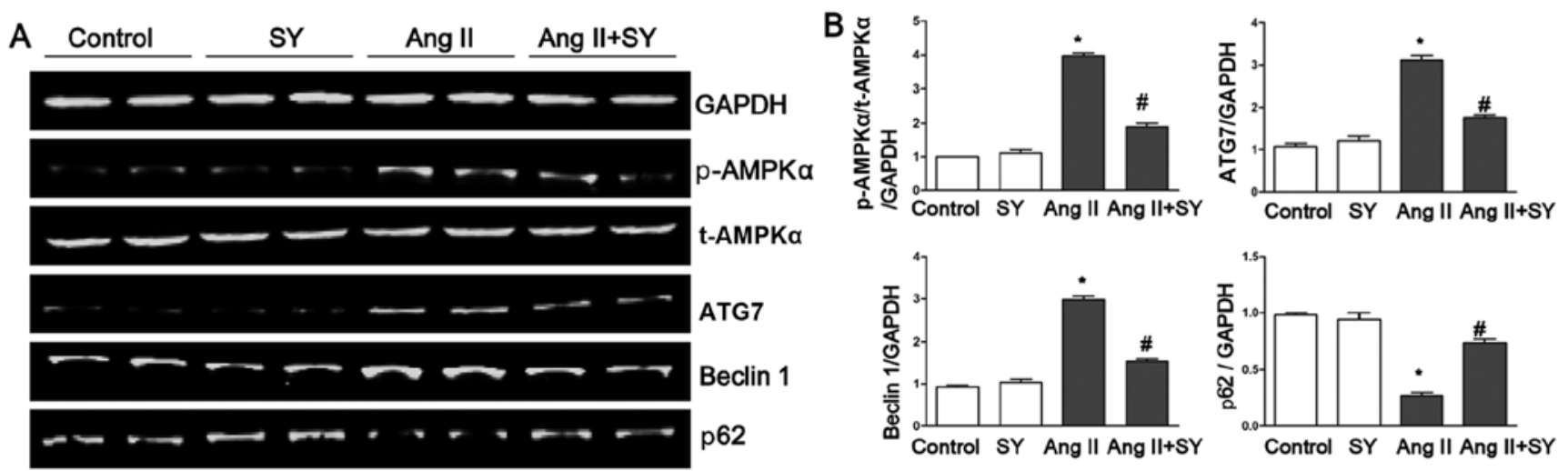

Figure 4. Syringin affects the AMP-activated protein kinase $\alpha$ (AMPK $\alpha$ ) and autophagy-related signaling pathways in H9c2 cells induced by Ang II (A) Representative blots of p-AMPK $\alpha$, ATG7, beclin 1 and $\mathrm{p} 62$ in $\mathrm{H} 9 \mathrm{c} 2$ cells. (B) Quantitative results. "p $<0.05$ compared with the control group; ${ }^{*} \mathrm{p}<0.05$ vs. the Ang II group. p, phosphorylation; t, total; SY, syringin $15 \mu \mathrm{M}$; Ra, rapamycin $100 \mathrm{nM}$.

Syringin is widely regarded as the major constituent of eleutherosides $(14,25)$. Over the years, this plant has been used extensively as a pharmacological agent to help the body to adapt to stress by supporting healthy adrenal gland function in many Asian countries $(13,16)$. In recent years, a number of studies have demonstrated that syringin promotes a wide range of biological activities. A previous study indicated that syringin injection at the desired doses $(100 \mathrm{~g} / \mathrm{kg})$ decreased plasma glucose and increased plasma insulin levels in fasted Wistar rats. This effect may be associated with the release of $\mathrm{ACh}$ from the nerve terminals, which in turn enhances insulin secretion (15). Another study by Niu et al indicated that in conscious rats with a regular sympathetic tone, the effect of syringin on plasma glucose regulation was impaired. Whereas in anesthetized animals, the decreased sympathetic tone may be helpful to the therapeutic benefits of syringin (16). Cui et al demonstrated that the administration of syringin decreased sleep latency and prolonged sleep duration, and that these effects may be mediated by the NOS/NO pathway (13). Another study indicated that syringin may alleviate the fulminant hepatic failure induced by lipopolysaccharide/D-galactosamine by attenuating NF- $\kappa \mathrm{B}$ activation to reduce inflammatory factor production, such as TNF- $\alpha$ (12). In conclusion, syringin promotes a wide range of biological activities. In this study, syringin attenuated the cardiac hypertrophyhic response induced by pressure overload and Ang II, as evidenced by changes in HW/BW, HW/TL and CSA in cardiomyocytes. Furthermore, our data indicated that syringin ameliorated LVEDd, IVSd, FS\%, EF\%, dP/ dt max and $\mathrm{dP} / \mathrm{dt}$ min which had been affected by AB. Thus, syringin attenuated cardiac dilation and improved LV function. Syringin also attenuated the increase in diastolic blood pressure and the decrease in systolic and diastolic functions. ANP and BNP, belong to the natriuretic peptides family, and are synthetized mainly in the heart (26). The expression levels of ANP and BNP, as in parallel with the degree of LV dysfunction and hemodynamic stress, have become the main index for the measurement of conventional cardiovascular disease risk factors and in parallel with the degree of LV dysfunction and hemodynamic stress (26-28). The present study demonstrated that syringin decreased the expression levels of ANP and BNP which were increased by $A B$, suggesting that syringin attenuated cardiac hypertrophy induced by pressure overload.
To further investigate the molecular mechanisms through which syringin attenuates cardiac hypertrophy, we examined activation of the AMPK $\alpha$ signaling pathway in response to stress stimuli. Our data demonstrated that syringin attenuated the phosphorylation of AMPK $\alpha$. Thus, we made the conjecture that syringing may play an important role in regulating the activation of AMPK $\alpha$. AMPK mediates energy metabolism by modulating the activities of key enzymes or their transcription factors in metabolic pathways (29-32) and also plays an important role in protein synthesis, myocyte apoptosis and myocardial angiogenesis. AMPK is composed of $\alpha, \beta$ and $\gamma$ subunits; each $\alpha$ subunit contains a phosphorylation site that plays a critical role in regulating AMPK function $(30,31)$. The specific role that AMPK $\alpha$ plays in myocardial metabolism remains incompletely understood. The study by Dyck and Lopaschuk demonstrated that AMPK $\alpha$ activation can influence cardiac metabolism by regulating oxidative phosphorylation and the uptake of fatty acids (33). Another study indicated that AMPK $\alpha$ was highly expressed in the embryonic stages and reduced to the adult level after birth; interestingly, in heart failure, the expression of AMPK $\alpha$ is increased $(30,34)$. Over the years, studies have demonstrated important roles of AMPK $\alpha$ in protecting the heart during ischemia/reperfusion injury, pathological hypertrophy and heart failure using both genetic and pharmacological approaches $(35,36)$. In a study on a model of ischemia/reperfusion, it was shown that AMPK clearly plays a cardioprotective role during ischemic episodes, by increasing glycolytic ATP production. However, AMPK can also play a deleterious role in the reperfused heart (37). Indeed, during early reperfusion, the still existing AMPK activation helps fatty acid oxidation to predominate over glucose oxidation by phosphorylating and inactivating acetyl-CoA carboxylase. Fatty acid oxidation, occurring in parallel with the still present glycolytic stimulation, can promote a deleterious uncoupling of glucose oxidation and glycolysis (38). This activation of AMPK results in the stimulation of glucose uptake, glycolysis and fatty acid oxidation $(32,34)$. These metabolic effects can be both beneficial and harmful during ischemia and during reperfusion following ischemia. In cardiac hypertrophy, AMPK activation can inhibit cardiac protein synthesis and the cardiac hypertrophic process $(33,39,40)$; however, on the other hand, both activating and inactivating AMPK mutations have been 
shown to contribute to cardiac hypertrophy (41-44). As our data demonstrated, it is possible that increased AMPK activity in the hypertrophic heart may actually be detrimental to cardiac function by accelerating fatty acid oxidation rates, such that they become the major oxidative substrate in the heart at the expense of glucose oxidation; syringin ameliorated the process. Furthermore, it has been proven that the anti-apoptotic and pro-apoptotic effects of AMPK, myocardial hypertrophy and ischemia can also induce apoptosis in the heart $(33,45,46)$. The role of AMPK in apoptosis or the role of apoptosis in myocardial hypertrophy is still not clear. It is possible that AMPK activation may play dual roles in the development of cardiac hypertrophy (33). That is, the pharmacological activation of AMPK during the early phase of cardiac hypertrophy may be able to prevent hypertrophic growth, while AMPK activation during pathological hypertrophy may be an adaptive response to the metabolic stress $(33,47,48)$. In addition, studies have elaborated that AMPK $\alpha$ plays an improtant role in cardiac hypertrophy by adjusting the process of autophagy (49-51). A previous study demonstrated that the elevation of autophagy during myocardial ischemia and glucose deprivation was accompanied by the activation of AMPK $\alpha$, and the inhibition of AMPK $\alpha$ significantly attenuated the induction of autophagy (52). In this study, syringin decreased hypertrophy induced by pressure overload by attenuating the activation of AMPK $\alpha$ and autophagy. The role of autophagy is complex, but is indispensable to maintain cardiac homeostasis $(22,49,53)$; it is difficult to confirm whether autophagy is protective or deleterious in the setting of cardiomyopathies $(53,54)$. The variationd in the expression of LC3II reveal that the level of autophagy may change in the different periods of left ventricular hypertrophy induced by transverse aortic constriction. Autophagy has been shown to be reduced in hypertrophied hearts following this type of surgery for 1 week, and elevated for 4 weeks $(55,56)$. Furthermore, our data indicated that cardiac hypertrophy induced by aortic banding involved AMPK-dependent autophagy. Another study indicated that autophagy was elevated in patient cardiac tissues affected by hypertrophic cardiomyopathy by detecting the expression of LC3II and beclin 1, which proved that miR-451 regulates cardiac hypertrophy and autophagy by targeting TSC1 (56). However, our data demonstrated that syringin reduced the autophagy level to alleviate cardiac hypertrophy. Our findings broaden our understanding of the protective role of syringing in cardiac hypertrophy, and provide a novel and potential pharmacotherapeutic strategy with which to mitigate cardiac hypertrophy induced by pressure overload and attenuate the progression of heart failure.

In conclusion, our data indicate that the long-term oral administration of syringin attenuates the development of cardiac hypertrophy induced by pressure overload and improves cardiac functions. The protective effects of syringin may potentially be attributed to the inhibition of the AMPK $\alpha$ and autophagy-related signaling pathways. Our results indicated the use of syringin may provide a potentially effective strategy with which to attenuate the progression of cardiac hypertrophy.

\section{Acknowledgements}

This study was supported by the Hubei Province Outstanding Medical Academic Leader program.

\section{References}

1. Wu QQ, Xu M, Yuan Y, Li FF, Yang Z, Liu Y, Zhou MQ, Bian ZY, Deng W, Gao L, et al: Cathepsin B deficiency attenuates cardiac remodeling in response to pressure overload via TNF- $\alpha /$ ASK1/JNK pathway. Am J Physiol Heart Circ Physiol 308: H1143-H1154, 2015.

2. Zhou H, Guo H, Zong J, Dai J, Yuan Y, Bian ZY and Tang QZ: ATF3 regulates multiple targets and may play a dual role in cardiac hypertrophy and injury. Int J Cardiol 174: 838-839, 2014.

3. Deng W, Zong J, Wei L, Guo H, Cheng Z, Zhang R, Lin Y and Tang Q: 3,3'-Diindolylmethane improves myocardial energy metabolism imbalance induced by pressure overload via AMPK $\alpha$ in mice. Int J Cardiol 177: 235-237, 2014.

4. Bian Z, Dai J, Hiroyasu N, Guan H, Yuan Y, Gan L, Zhou H, Zong J, Zhang Y, Li F, et al: Disruption of tumor necrosis factor receptor associated factor 5 exacerbates pressure overload cardiac hypertrophy and fibrosis. J Cell Biochem 115: 349-358, 2014.

5. Zong J, Wu QQ, Zhou H, Zhang JY, Yuan Y, Bian ZY, Deng W, Dai J, Li FF, Xu M, et al: 3,3'-Diindolylmethane attenuates cardiac $\mathrm{H} 9 \mathrm{c} 2$ cell hypertrophy through 5'-adenosine monophosphate-activated protein kinase- $\alpha$. Mol Med Rep 12: 1247-1252, 2015.

6. Zhou H, Yuan Y, Liu Y, Ni J, Deng W, Bian ZY, Dai J and Tang QZ: Icariin protects H9c2 cardiomyocytes from lipopolysaccharide induced injury via inhibition of the reactive oxygen species dependent $\mathrm{c}$ Jun $\mathrm{N}$ terminal kinases/nuclear factor- $\mathrm{kB}$ pathway. Mol Med Rep 11: 4327-4332, 2015.

7. Deng W, Wang X, Xiao J, Chen K, Zhou H, Shen D, Li H and Tang Q: Loss of regulator of $\mathrm{G}$ protein signaling 5 exacerbates obesity, hepatic steatosis, inflammation and insulin resistance. PLoS One 7: e30256, 2012.

8. Zong J, Salim M, Zhou H, Bian ZY, Dai J, Yuan Y, Deng W, Zhang JY, Zhang R, Wu QQ and Tang QZ: NOD2 deletion promotes cardiac hypertrophy and fibrosis induced by pressure overload. Lab Invest 93: 1128-1136, 2013.

9. Yussman MG, Toyokawa T, Odley A, Lynch RA, Wu G, Colbert MC, Aronow BJ, Lorenz JN and Dorn GW II: Mitochondrial death protein Nix is induced in cardiac hypertrophy and triggers apoptotic cardiomyopathy. Nat Med 8: 725-730, 2002

10. Fernandes RO, Dreher GJ, Schenkel PC, Fernandes TR, Ribeiro MF, Araujo AS and Belló-Klein A: Redox status and pro-survival/pro-apoptotic protein expression in the early cardiac hypertrophy induced by experimental hyperthyroidism. Cell Biochem Funct 29: 617-623, 2011.

11. Adams JW, Sakata Y, Davis MG, Sah VP, Wang Y, Liggett SB, Chien KR, Brown JH and Dorn GW II: Enhanced Galphaq signaling: A common pathway mediates cardiac hypertrophy and apoptotic heart failure. Proc Natl Acad Sci USA 95: 10140-10145, 1998.

12. Gong X, Zhang L, Jiang R, Wang CD, Yin XR and Wan JY: Hepatoprotective effects of syringin on fulminant hepatic failure induced by $\mathrm{D}$-galactosamine and lipopolysaccharide in mice. J Appl Toxicol 34: 265-271, 2014.

13. Cui Y, Zhang Y and Liu G: Syringin may exert sleep-potentiating effects through the NOS/NO pathway. Fundam Clin Pharmacol 29: 178-184, 2015.

14. Li Q, Sun LX, Xu L, Jia Y, Wang ZW, Shen ZD and Bi KS: Determination and pharmacokinetic study of syringin and chlorogenic acid in rat plasma after administration of Aidi lyophilizer. Biomed Chromatogr 20: 1315-1320, 2006.

15. Liu KY, Wu YC, Liu IM, Yu WC and Cheng JT: Release of acetylcholine by syringin, an active principle of Eleutherococcus senticosus, to raise insulin secretion in Wistar rats. Neurosci Lett 434: 195-199, 2008.

16. Niu HS, Hsu FL and Liu IM: Role of sympathetic tone in the loss of syringin-induced plasma glucose lowering action in conscious Wistar rats. Neurosci Lett 445: 113-116, 2008.

17. Cho JY, Nam KH, Kim AR, Park J, Yoo ES, Baik KU, Yu YH and Park MH: In-vitro and in-vivo immunomodulatory effects of syringin. J Pharm Pharmacol 53: 1287-1294, 2001.

18. Yan L, Vatner DE, Kim SJ, Ge H, Masurekar M, Massover WH, Yang G, Matsui Y, Sadoshima J and Vatner SF: Autophagy in chronically ischemic myocardium. Proc Natl Acad Sci USA 102: 13807-13812, 2005. 
19. Godar RJ, Ma X, Liu H, Murphy JT, Weinheimer CJ, Kovacs A, Crosby SD, Saftig P and Diwan A: Repetitive stimulation of autophagy-lysosome machinery by intermittent fasting preconditions the myocardium to ischemia-reperfusion injury. Autophagy 11: 1537-1560, 2015.

20. Higashi K, Yamada Y, Minatoguchi S, Baba S, Iwasa M, Kanamori H, Kawasaki M, Nishigaki $K$, Takemura G, Kumazaki M, et al: MicroRNA-145 repairs infarcted myocardium by accelerating cardiomyocyte autophagy. Am J Physiol Heart Circ Physiol 309: H1813-H1826, 2015.

21. Zhu H, Tannous P, Johnstone JL, Kong Y, Shelton JM, Richardson JA, Le V, Levine B, Rothermel BA and Hill JA: Cardiac autophagy is a maladaptive response to hemodynamic stress. J Clin Invest 117: 1782-1793, 2007.

22. Li Z, Wang J and Yang X: Functions of autophagy in pathological cardiac hypertrophy. Int J Biol Sci 11: 672-678, 2015.

23. Xu X, Hua Y, Nair S, Bucala R and Ren J: Macrophage migration inhibitory factor deletion exacerbates pressure overloadinduced cardiac hypertrophy through mitigating autophagy. Hypertension 63: 490-499, 2014

24. Tohda C, Ichimura M, Bai Y, Tanaka K, Zhu S and Komatsu K Inhibitory effects of Eleutherococcus senticosus extracts on amyloid $\beta(25-35)$-induced neuritic atrophy and synaptic loss. J Pharmacol Sci 107: 329-339, 2008

25. Ma B, Zhang Q, Liu Y, Li J, Xu Q, Li X, Yang X, Yao D, Sun J, Cui G, et al: Simultaneous determination of Eleutheroside B and Eleutheroside $\mathrm{E}$ in rat plasma by high performance liquid chromatography-electrospray ionization mass spectrometry and its application in a pharmacokinetic study. J Chromatogr B Analyt Technol Biomed Life Sci 917-918: 84-92, 2013.

26. Sergeeva IA and Christoffels VM: Regulation of expression of atrial and brain natriuretic peptide, biomarkers for heart development and disease. Biochim Biophys Acta 1832: 2403-2413, 2013.

27. Wu Q, Xu-Cai YO, Chen S and Wang W: Corin: New insights into the natriuretic peptide system. Kidney Int 75: 142-146, 2009.

28. Volpe M, Rubattu S and Burnett J Jr: Natriuretic peptides in cardiovascular diseases: Current use and perspectives. Eur Heart J 35: 419-425, 2014.

29. Zhang Y, Mi SL, Hu N, Doser TA, Sun A, Ge J and Ren J: Mitochondrial aldehyde dehydrogenase 2 accentuates aginginduced cardiac remodeling and contractile dysfunction: Role of AMPK, Sirt1, and mitochondrial function. Free Radic Biol Med 71: 208-220, 2014

30. Kim M, Shen M, Ngoy S, Karamanlidis G, Liao R and Tian R AMPK isoform expression in the normal and failing hearts. J Mol Cell Cardiol 52: 1066-1073, 2012.

31. Turdi S, Kandadi MR, Zhao J, Huff AF, Du M and Ren J: Deficiency in AMP-activated protein kinase exaggerates high fat diet-induced cardiac hypertrophy and contractile dysfunction. J Mol Cell Cardiol 50: 712-722, 2011.

32. Beauloye C, Bertrand L, Horman S and Hue L: AMPK activation, a preventive therapeutic target in the transition from cardiac injury to heart failure. Cardiovasc Res 90: 224-233, 2011.

33. Dyck JR and Lopaschuk GD: AMPK alterations in cardiac physiology and pathology: Enemy or ally? J Physiol 574: 95-112 2006

34. Meng R, Pei Z, Zhang A, Zhou Y, Cai X, Chen B, Liu G, Mai W, Wei J and Dong Y: AMPK activation enhances PPAR $\alpha$ activity to inhibit cardiac hypertrophy via ERK1/2 MAPK signaling pathway. Arch Biochem Biophys 511: 1-7, 2011.

35. Li Y, Chen C, Yao F, Su Q, Liu D, Xue R, Dai G, Fang R, Zeng J, Chen Y, et al: AMPK inhibits cardiac hypertrophy by promoting autophagy via mTORC1. Arch Biochem Biophys 558: 79-86, 2014.

36. Kudo N, Barr AJ, Barr RL, Desai S and Lopaschuk GD: High rates of fatty acid oxidation during reperfusion of ischemic hearts are associated with a decrease in malonyl-CoA levels due to an increase in 5'-AMP-activated protein kinase inhibition of acetyl-CoA carboxylase. J Biol Chem 270: 17513-17520, 1995.

37. Kudo N, Gillespie JG, Kung L, Witters LA, Schulz R, Clanachan AS and Lopaschuk GD: Characterization of 5'AMP-activated protein kinase activity in the heart and its role in inhibiting acetyl-CoA carboxylase during reperfusion following ischemia. Biochim Biophys Acta 1301: 67-75, 1996.

38. Fassett JT, Hu X, Xu X, Lu Z, Zhang P, Chen Y and Bache RJ: AMPK attenuates microtubule proliferation in cardiac hypertrophy. Am J Physiol Heart Circ Physiol 304: H749-H758, 2013
39. Zhu J, Ning RB, Lin XY, Chai DJ, Xu CS, Xie H, Zeng JZ and Lin JX: Retinoid X receptor agonists inhibit hypertension-induced myocardial hypertrophy by modulating LKB1/AMPK/p70S6K signaling pathway. Am J Hypertens 27: 1112-1124, 2014.

40. Davies JK, Wells DJ, Liu K, Whitrow HR, Daniel TD, Grignani R, Lygate CA, Schneider JE, Noël G, Watkins H and Carling D: Characterization of the role of gamma2 R531G mutation in AMP-activated protein kinase in cardiac hypertrophy and WolffParkinson-White syndrome. Am J Physiol Heart Circ Physiol 290: H1942-H1951, 2006.

41. Patel VV, Arad M, Moskowitz IP, Maguire CT, Branco D, Seidman JG, Seidman CE and Berul CI: Electrophysiologic characterization and postnatal development of ventricular pre-excitation in a mouse model of cardiac hypertrophy and Wolff-Parkinson-White syndrome. J Am Coll Cardiol 42: 942-951, 2003.

42. Arad M, Moskowitz IP, Patel VV, Ahmad F, Perez-Atayde AR, Sawyer DB, Walter M, Li GH, Burgon PG, Maguire CT, et al: Transgenic mice overexpressing mutant PRKAG2 define the cause of Wolff-Parkinson-White syndrome in glycogen storage cardiomyopathy. Circulation 107: 2850-2856, 2003.

43. Gollob MH, Green MS, Tang AS, Gollob T, Karibe A, Ali Hassan AS, Ahmad F, Lozado R, Shah G, Fananapazir L, et al: Identification of a gene responsible for familial WolffParkinson-White syndrome. N Engl J Med 344: 1823-1831, 2001.

44. Capano $\mathrm{M}$ and Crompton M: Bax translocates to mitochondria of heart cells during simulated ischaemia: Involvement of AMP-activated and p38 mitogen-activated protein kinases. Biochem J 395: 57-64, 2006.

45. Russell RR III, Li J, Coven DL, Pypaert M, Zechner C, Palmeri M, Giordano FJ, Mu J, Birnbaum MJ and Young LH: AMP-activated protein kinase mediates ischemic glucose uptake and prevents postischemic cardiac dysfunction, apoptosis, and injury. J Clin Invest 114: 495-503, 2004.

46. Nagata $D$ and Hirata $Y$ : The role of AMP-activated protein kinase in the cardiovascular system. Hypertens Res 33: 22-28, 2010.

47. Horman S, Beauloye C, Vanoverschelde JL and Bertrand L: AMP-activated protein kinase in the control of cardiac metabolism and remodeling. Curr Heart Fail Rep 9: 164-173, 2012.

48. Xie S, Deng Y, Pan YY, Wang ZH, Ren J, Guo XL, Yuan X, Shang $\mathrm{J}$ and Liu HG: Melatonin protects against chronic intermittent hypoxia-induced cardiac hypertrophy by modulating autophagy through the 5' adenosine monophosphate-activated protein kinase pathway. Biochem Biophys Res Commun 464: 975-981, 2015.

49. Liu L, Wang C, Sun D, Jiang S, Li H, Zhang W, Zhao Y, Xi Y, Shi S, Lu F, et al: Calhex 231 ameliorates cardiac hypertrophy by inhibiting cellular autophagy in vivo and in vitro. Cell Physiol Biochem 36: 1597-1612, 2015.

50. Liu B, Wu Z, Li Y, Ou C, Huang Z, Zhang J, Liu P, Luo C and Chen $M$ : Puerarin prevents cardiac hypertrophy induced by pressure overload through activation of autophagy. Biochem Biophys Res Commun 464: 908-915, 2015.

51. Mellor KM, Reichelt ME and Delbridge LM: Autophagy anomalies in the diabetic myocardium. Autophagy 7: 1263-1267, 2011.

52. Zhang X, Gibson ME, Li ZL, Zhu XY, Jordan KL, Lerman A and Lerman LO: Autophagy portends the level of cardiac hypertrophy in experimental hypertensive swine model. Am J Hypertens 29: 81-89, 2016.

53. Yu P, Zhang Y, Li C, Li Y, Jiang S, Zhang X, Ding Z, Tu F, Wu J, Gao X and Li L: Class III PI3K-mediated prolonged activation of autophagy plays a critical role in the transition of cardiac hypertrophy to heart failure. J Cell Mol Med 19: 1710-1719, 2015.

54. Oyabu J, Yamaguchi O, Hikoso S, Takeda T, Oka T, Murakawa T, Yasui $\mathrm{H}$, Ueda H, Nakayama H, Taneike M, et al: Autophagymediated degradation is necessary for regression of cardiac hypertrophy during ventricular unloading. Biochem Biophys Res Commun 441: 787-792, 2013

55. Hariharan N, Ikeda Y, Hong C, Alcendor RR, Usui S, Gao S, Maejima Y and Sadoshima J: Autophagy plays an essential role in mediating regression of hypertrophy during unloading of the heart. PLoS One 8: e51632, 2013

56. Song L, Su M, Wang S, Zou Y, Wang X, Wang Y, Cui H, Zhao P, Hui R and Wang J: MiR-451 is decreased in hypertrophic cardiomyopathy and regulates autophagy by targeting TSC1. J Cell Mol Med 18: 2266-2274, 2014. 